1998-11-01

\title{
Monte Carlo simulation of an ion-dipole mixture as a model of an electrical double layer
}

Douglas Henderson

Dezso Boda

Kwong-Yu Chan

Follow this and additional works at: https://scholarsarchive.byu.edu/facpub

Part of the Biochemistry Commons, and the Chemistry Commons

\section{Original Publication Citation}

Boda, Dezso, Kwong Y. Chan, and Douglas Henderson."Monte Carlo simulation of an ion-dipole mixture as a model of an electrical double layer." The Journal of Chemical Physics 19 (1998): 7362-7371.

\section{BYU ScholarsArchive Citation}

Henderson, Douglas; Boda, Dezso; and Chan, Kwong-Yu, "Monte Carlo simulation of an ion-dipole mixture as a model of an electrical double layer" (1998). Faculty Publications. 635.

https://scholarsarchive.byu.edu/facpub/635 


\title{
Monte Carlo simulation of an ion-dipole mixture as a model of an electrical double layer
}

\author{
Dezsö Boda ${ }^{\mathrm{a})}$ and Kwong-Yu Chan ${ }^{\mathrm{b})}$ \\ Department of Chemistry, The University of Hong Kong, Pokfulam Road, Hong Kong \\ Douglas Henderson ${ }^{c}$ \\ Department of Chemistry and Biochemistry, Brigham Young University, Provo, Utah 84602-5700
}

(Received 14 May 1998; accepted 29 July 1998)

\begin{abstract}
Canonical Monte Carlo simulations were performed for a nonprimitive model of an electrical double layer. The ions and the solvent molecules are modeled as charged and dipolar hard spheres, respectively, while the electrode as a hard, impenetrable wall carrying uniform surface charge. We found that the ion-dipole model gives a reasonable description of the double layer for partially charged ions with small to moderate dipole moments, or equivalently for an "effective" dielectric constant. Density, polarization and mean electrostatic potential profiles are reported. Strong layering structure, and at higher charges, charge inversion in the second layer were found. With appropriate choices of charge and solvent parameters, states corresponding to the primitive or the solvent primitive model can be produced, and the results agreed well with literature data. At higher effective charges and dipole moments, the dipolar solvent has difficulties in preventing the ions from clustering. More realistic models of water and other solvents are necessary to study the double layer. (C) 1998 American Institute of Physics. [S0021-9606(98)51341-4]
\end{abstract}

\section{INTRODUCTION}

The electrochemical double layer (DL) has great importance in electrochemical, biological, colloidal and interface sciences. It has been studied widely using the primitive model (PM), where the ions are represented as charged hard spheres, the solvent as an isotropic dielectric continuum, and the surface as a hard wall with a uniform surface charge. This model was investigated by integral equation ${ }^{1-5}$ and density functional $(\mathrm{DF})^{6-8}$ theories, as well as computer simulations in the canonical ${ }^{9-11}$ and the grand canonical ${ }^{12-16}$ ensembles. The results showed good agreement with the classical Gouy-Chapman theory for low surface charges and 1:1 electrolyte solutions of moderate concentrations. Differences were noted, however, for higher valence salts and/or high surface charge densities.

The basic disadvantage of the PM is that it ignores the molecular nature of the solvent. To take into consideration the effect of the solvent molecules and to examine the solvent structure at the interface, more realistic models were developed (often called nonprimitive or "civilized" models), where both the ions and the solvent are treated on a molecular basis. One of the simplest models is the ion-dipole mixture where the solvent molecules are modeled as hard spheres with embedded point dipoles. This was examined using the mean spherical approximation (MSA). ${ }^{17,18}$ Another more sophisticated nonprimitive model, where the solvent particles have, in addition to the point dipole, a quadrupole

${ }^{a)}$ Permanent address: Department of Physical Chemistry, University of Veszprém, H-8201 Veszprém, P.O. Box 158, Hungary. Electronic mail: boda@almos.vein.hu

${ }^{b}$ Electronic mail: hrsccky@hkucc.hku.hk

${ }^{c}$ Electronic mail: doug@huey.byu.edu tensor describing the water molecule, was investigated by the reference hypernetted-chain (RHNC) theory. ${ }^{19}$ Both MSA and RHNC calculations have revealed the importance of the solvent structure in the DL. Oscillatory behavior and directional ordering near the charged surface were found.

To investigate more sophisticated nonprimitive models of the electrical double layer, computer simulations appear to be reasonable, with the development of modern computers and increasingly powerful methods. Although to our knowledge, for the confined ion-dipole mixture model no simulation data have been published, recently a more realistic DL model was studied by $\mathrm{Spohr}^{20}$ with the molecular dynamics (MD) simulation method. He investigated aqueous $\mathrm{NaCl}$ and $\mathrm{CsF}$ solutions near a model electrode. The ions were modeled as charged Lennard-Jones (LJ) particles, and the water molecules were described by the rigid SPC/E model. The electrode potential, besides the electrostatic and imagecharge interactions, contains a nonelectrostatic contribution describing the effect of surface corrugation and anisotropic adsorption. Spohr's investigations showed different behavior for the various ions. For instance, the smaller ions tend to form rigid solvation shells that prevented them from contact adsorption (Glosli and Philpott, ${ }^{21}$ studying a similar system, observed the same behavior); while the larger ones show stronger contact adsorption. Contact adsorption did not occur on the uncharged electrodes.

In the case of the ion-dipole mixture the electrostatic forces dominate the system, and the short range interactions are represented by the hard exclusions. This model addresses the basic features of the DL structure that are driven by the electrostatic attractions and repulsions, and it can reveal the importance of the solvent structure with respect to simpler models (e.g., the PM). The computer simulation of ion- 
dipole mixtures appears to be technically difficult even in the bulk fluid state. ${ }^{22-27}$ The ion-dipole mixture has many low energy configurations separated by strong barriers. Examples of such minima are ion-pairing, a fully solvated ion, and, in the case of the DL system, layering at the charged surface. The standard Metropolis MC technique is slow to move the system out of relative minima and the sampling is very inefficient. In previous bulk simulations $\mathrm{s}^{22-25}$ hundreds of millions MC moves were needed to break away from a local minimum.

To avoid some of these problems, Davis et al..$^{28-30}$ used a simplified model in which the solvent molecules are neutral hard spheres and their polar nature was taken into account by the screening in the continuum model with $\epsilon=78.5$. This model was used earlier by Henderson and Lozada-Cassou ${ }^{4}$ to explain hydration forces in colloidal suspensions. Davis et al. have studied this model, which they call the solvent primitive model (SPM), by DF theory ${ }^{28,29}$ and Monte Carlo (MC) simulation. ${ }^{30}$ Their investigations showed, in agreement with Henderson and Lozada-Cassou, that the presence of the solvent molecules induced strong structures, as evidenced by the ion and solvent distributions. Several layers of ions and solvent particles were found near both the charged and the neutral walls. These features are absent in the PM simulations.

Although the SPM avoids the difficulties due to the polar nature of the solvent molecules, their treatment as particles of a finite size results in a system with a high density. This makes the calculation of the chemical potential problematic because particle insertions, in grand canonical MC (GCMC) simulations or test particle techniques with efficient sampling, are difficult to implement. Without the chemical potential, the correspondence between the confined system and bulk electrolyte of a given concentration, and thus comparison with results of theoretical methods, cannot be made precisely.

Zhang et al. ${ }^{30}$ proposed a trick to evade this problem. They performed GCMC simulations in both bulk and confined systems for the PM model, and obtained the bulk concentration and the average numbers of ions in the DL system, respectively, for a given value of the electrochemical potential. Then, they added neutral hard spheres, as solvent molecules, to the ions until the density reached a given liquidlike value and canonical MC simulations for this SPM system were performed. Their procedure obviously lacks consistency since adding hard spheres changes the chemical potentials as has been shown by HNC calculations ${ }^{31}$ and simulations. ${ }^{32}$ Nevertheless, it seems a reasonable approximation because Zhang et al. ${ }^{30}$ obtained back the estimated bulk density in the middle of the cell. It can be expected that in the case of the ion-dipole mixture, this approximation will be less satisfactory; but in the absence of reasonable GCMC data, it can be accepted as a starting point for this preliminary investigation.

Thus, one of the state points of Zhang et al. ${ }^{30}$ was chosen to be a basic state of our investigations. For the new state points we used a temperature of $300 \mathrm{~K}$ and the dielectric constant of 1 . The charges of the ions are $\pm e$, the dipole moment of the solvent molecules is 1.8 Debye, and for the diameter of the particles the more waterlike $3 \AA$ was used instead of the $4.25 \AA$ of Zhang et al. The choice of the hard core diameter of $4.25 \AA$ seems to come from the early studies of the PM and it is too large for water and many ions. We believe that in studies of nonprimitive models, a diameter representing water, and that is not unrealistic for common ions, is more logical.

We found that with the above parameters efficient simulations cannot be performed. Because of the oversimplified treatment of the nonelectrostatic parts of the potentials, the dominating long range electrostatic parts produce too deep local potential wells in the phase space, and the particles tend to form low-energy configurations from which they cannot be moved out in a reasonable length of simulation. This means that the system is "practically nonergodic" in the sense of Larsen and Rogde. ${ }^{33}$ Therefore, it became necessary to moderate the strengths of the charges and/or the dipoles. For this purpose we introduced a "charging" parameter $\lambda_{i}$ and a "polarizing" parameter $\lambda_{\mu}$. By multiplying the charge by $\lambda_{i}$ and the dipole moment by $\lambda_{\mu}$, we can modulate the strengths of the ionic and dipolar interactions. The $\lambda$ 's can be thought to be a measure of an "effective" dielectric constant. For example, $\lambda$ 's in the range 0.2 to 0.5 correspond to an "effective" dielectric constant between about 25 and 4.

Canonical simulations were performed for some appropriate values of the parameters with both charged and uncharged walls. The density and potential profiles that we obtain show different behavior in the $\lambda$ range that we consider, and imply an even more structured interfacial region than that was obtained from the SPM simulations. We have developed a method for treating long range corrections of the ion-dipole mixture in a confined geometry that is described in the next section. To test the simulation method, simulations were performed for the PM and SPM systems, and the results were compared with those of Torrie and Valleau ${ }^{13}$ and of Zhang et al. ${ }^{30}$ Further tests were made by simulating a pure DHS fluid confined between charged and uncharged walls. In the last section we discuss our results, which are believed to provide a contribution to the better understanding the structure of DL systems.

\section{MODEL}

Consider a mixture of $N_{+}$cations with charge $q_{+}$and HS particle diameter $d_{+}, N_{-}$anions with charge $q_{-}$and diameter $d_{-}$, and $N_{\mu}$ dipoles of dipole moment $\mu$ and diameter $d_{\mu}$ at a temperature $T$. The particles are confined in a rectangular simulation cell whose dimensions are $W \times W$ $\times H$, with hard impenetrable walls at $z=0$ and $z=H$, while periodic boundary conditions are applied in the $x$ and $y$ directions. The left and right walls carry uniform surface charge densities $\sigma_{1}$ and $\sigma_{2}$, respectively. We require that $\left(\sigma_{1}+\sigma_{2}\right) W^{2}=-\left(N_{+} q_{+}+N_{-} q_{-}\right)$so that the system is electrostatically neutral.

The potential energy of the system is a sum of oneparticle and two-particle energies. The pair interactions acting between the various species of molecules are the wellknown multipole potentials with the corresponding hard sphere (HS) repulsive cores. They can be found in Eqs. (7)- 
(9) below. But first some details about the treatment of the long range corrections should be given. Our method is based on that introduced by Torrie and Valleau. ${ }^{13}$ The influence of the lateral charges and dipoles surrounding the central cell is taken into account by infinite sheets parallel to the charged walls. In our algorithm, each ion and dipole has its own sheet at the same $z$ coordinate as the particle. Charged sheets correspond to each ion and polarized sheets to each dipole. A charged sheet carries a uniform charge density of $q / W^{2}$ where $q$ is the charge of the central ion, while a polarized sheet has a uniform surface polarization density of $\boldsymbol{\mu} / W^{2}$ where $\boldsymbol{\mu}$ is the dipole moment of the central dipole. Each particle interacts with each sheet, less the square "hole", corresponding to the central particle in which the interaction is taken into account explicitly by the intermolecular potentials. The potential energy of an ion $q_{i}$ above the center of a charged sheet ( $c s h)$ of dimension $L \times L$ corresponding to an ion $q_{j}$ is

$$
\begin{aligned}
u_{i, c s h}\left(z_{i}, z_{j}, L\right) & =q_{i} \frac{q_{j}}{L^{2}} \int_{-L / 2}^{L / 2} \int_{-L / 2}^{L / 2} \frac{d x d y}{r} \\
& =q_{i} \frac{q_{j}}{L^{2}} \phi(z, L),
\end{aligned}
$$

between the ion $q_{i}$ and a polarized sheet ( $p s h$ ) corresponding to a dipole $\boldsymbol{\mu}_{j}$ at $z_{j}$ is

$$
\begin{aligned}
u_{i, p s h}\left(z_{i}, z_{j}, \mu_{j, z}, L\right) & =q_{i} \frac{1}{L^{2}} \int_{-L / 2}^{L / 2} \int_{-L / 2}^{L / 2} \frac{\mathbf{r} \cdot \boldsymbol{\mu}_{j}}{r^{3}} d x d y \\
& =\operatorname{sign}(z) q_{i} \frac{\mu_{j, z}}{L^{2}} E(z, L),
\end{aligned}
$$

and between the dipole $\boldsymbol{\mu}_{i}$ and a polarized sheet corresponding to dipole $\boldsymbol{\mu}_{j}$ is

$$
\begin{aligned}
u_{\mu, p s h}\left(z_{i}, z_{j}, \boldsymbol{\mu}_{i}, \boldsymbol{\mu}_{j}, L\right) & \\
= & -\frac{1}{L^{2}} \int_{-L / 2}^{L / 2} \int_{-L / 2}^{L / 2}\left[\frac{3\left(\mathbf{r} \cdot \boldsymbol{\mu}_{i}\right)\left(\mathbf{r} \cdot \boldsymbol{\mu}_{j}\right)}{r^{5}}-\frac{\boldsymbol{\mu}_{i} \cdot \boldsymbol{\mu}_{j}}{r^{3}}\right] d x d y \\
& -\left(3 \mu_{i, z} \frac{\mu_{j, z}}{L^{2}}-\boldsymbol{\mu}_{i} \cdot \frac{\boldsymbol{\mu}_{j}}{L^{2}}\right) \Delta(z, L),
\end{aligned}
$$

where the vector $\mathbf{r}=\left(x, y, z_{i}-z_{j}\right)$ points from the unit area $d x d y$ of the sheet to the particle, $|z|=\left|z_{i}-z_{j}\right|$ is the distance between a particle and a sheet, and the functions in Eqs. (1)-(3) by performing the integrations can be expressed as

$$
\begin{aligned}
& E(z, L)=2 \pi-4 \arctan \frac{4|z| r_{1}}{L}, \\
& \phi(z, L)=4 L \ln \left(\frac{0.5+r_{1}}{r_{2}}\right)-|z| E(z, L),
\end{aligned}
$$

and

$$
\Delta(z, L)=\frac{1}{L r_{1} r_{2}^{2}},
$$

with $r_{1}=\sqrt{0.5+(z / L)^{2}}$ and $r_{2}=\sqrt{0.25+(z / L)^{2}}$. Because of symmetry, the dipole-charged sheet interaction is equal to the ion-polarized sheet interaction: $u_{\mu, c s h}=u_{i, p s h}$.
To save computer time, it is worth using infinite sheets. The values of the functions in Eqs. (1)-(3) in the limit of $L \rightarrow \infty$ are $E(z, \infty)=2 \pi, \phi(z, \infty)=-2 \pi|z|$ and $\Delta(z, \infty)=0$. The divergent first term of $\phi$ vanishes because of the charge neutrality of the system. Thus, the total two-particle energies (the ion-ion, the ion-dipole and the dipole-dipole terms) of the system can be expressed as

$$
\begin{aligned}
U_{i i}= & \sum_{i=1}^{N_{i}-1} \sum_{j=i+1}^{N_{i}}\left[u_{\mathrm{HS}}\left(d_{i j}, r_{i j}\right)+\frac{q_{i} q_{j}}{r_{i j}}\right] \\
& +\sum_{i=1}^{N_{i}} \sum_{j=1}^{N_{i}}\left[u_{i, c s h}(i, j, \infty)-u_{i, c s h}(i, j, W)\right], \\
U_{i \mu}= & \sum_{i=1}^{N_{i}} \sum_{j=1}^{N_{\mu}}\left[u_{\mathrm{HS}}\left(d_{i j}, r_{i j}\right)\right. \\
& \left.+q_{i} \frac{\boldsymbol{\mu}_{i} \cdot \mathbf{r}_{i j}}{r_{i j}^{3}}+u_{i, p s h}(i, j, \infty)-u_{i, p s h}(i, j, W)\right],
\end{aligned}
$$

and

$$
\begin{aligned}
U_{\mu \mu}= & \sum_{i=1}^{N_{\mu}-1} \sum_{j=i+1}^{N_{\mu}}\left[u_{\mathrm{HS}}\left(d_{\mu}, r_{i j}\right)\right. \\
& \left.-\frac{3\left(\boldsymbol{\mu}_{i} \cdot \mathbf{r}_{i j}\right)\left(\boldsymbol{\mu}_{i} \cdot \mathbf{r}_{i j}\right)}{r_{i j}^{5}}+\frac{\boldsymbol{\mu}_{i} \cdot \boldsymbol{\mu}_{j}}{r_{i j}^{3}}\right] \\
& +\sum_{i=1}^{N_{\mu}} \sum_{j=1}^{N_{\mu}}\left[u_{d, p s h}(i, j, \infty)-u_{d, p s h}(i, j, W)\right],
\end{aligned}
$$

where $N_{i}=N_{+}+N_{-}$is the number of ions, $\mathbf{r}_{i j}=\mathbf{r}_{i}-\mathbf{r}_{j}, r_{i j}$ $=\left|\mathbf{r}_{i j}\right|$ is the distance while $d_{i j}=\left(d_{i}+d_{j}\right) / 2$ is the distance in touch of the corresponding particles, $(i, j, W)$ is the brief notation for the argument of the corresponding functions. Finally,

$$
u_{\mathrm{HS}}\left(d, r_{i j}\right)= \begin{cases}\infty & \text { if } r_{i j}<d \\ 0 & \text { otherwise }\end{cases}
$$

is the hard-sphere (HS) interaction.

The one-particle energies (ion-wall, dipole-wall) with the infinite charged hard walls $(w)$ are

$$
\begin{aligned}
U_{i w}= & \sum_{i=1}^{N_{i}}\left[u_{\mathrm{HS}}\left(d_{i} / 2, z_{i}\right)+u_{\mathrm{HS}}\left(d_{i} / 2, H-z_{i}\right)\right. \\
& \left.-2 \pi q_{i} z_{i} \sigma_{1}-2 \pi q_{i}\left(H-z_{i}\right) \sigma_{2}\right]
\end{aligned}
$$

and

$$
\begin{aligned}
U_{\mu w}= & \sum_{i=1}^{N_{\mu}}\left[u_{\mathrm{HS}}\left(d_{\mu} / 2, z_{i}\right)+u_{\mathrm{HS}}\left(d_{\mu} / 2, H-z_{i}\right)\right. \\
& \left.-2 \pi \mu_{i, z} \sigma_{1}+2 \pi \mu_{i, z} \sigma_{2}\right] .
\end{aligned}
$$

Note that Torrie and Valleau ${ }^{13}$ used equidistantly spaced sheets carrying surface charge densities corresponding to an averaged charge distribution in the central cell. This procedure includes information from earlier stages of the system, and thus, the simulation is not rigorously a Markov-chain. In our simulation this is not the case because the sheets are continuously moving with the particles and the energy 
TABLE I. Parameters of the simulations. The temperature is $T=300 \mathrm{~K}$, the charges of the ions are $q_{ \pm}= \pm \lambda_{i} e$, the dipole moment strength of the solvent molecules is $\mu=\lambda_{\mu} \times 1.8$ Debye, the diameter of the particles are $d=3 \AA$ except for simulations $a$ and $b$, where $4.25 \AA$ was used. The reduced surface charge is $\sigma^{*}=\sigma_{1} d^{2} / e$ on the left wall, the right wall is neutral except for simulations $c-f$ where $\sigma_{2}=-\sigma_{1}$. The column "Fig." shows in which figure the density profiles of the corresponding simulation can be seen. The length of the simulations is 4 million MC steps after equilibration.

\begin{tabular}{|c|c|c|c|c|c|c|c|c|c|c|c|c|}
\hline & $-\sigma^{*}$ & $\lambda_{i}$ & $\lambda_{\mu}$ & $N_{+}$ & $N_{-}$ & $N_{\mu}$ & $W / d$ & $H / d$ & $n_{i, 0}$ & $n_{\mu, 0}$ & Fig. & Note \\
\hline $\mathrm{a}$ & 0.3 & 0.113 & - & 234 & 54 & - & 24.5 & 24.5 & 0.0044 & - & 1(a) & $\mathrm{PM}$ \\
\hline $\mathrm{b}$ & 0.42 & 0.113 & 0 & 17 & 9 & 134 & 4.36 & 12 & 0.045 & 0.627 & $1(b)$ & SPM \\
\hline $\mathrm{c}$ & 0.0 & \multirow{4}{*}{-} & \multirow{4}{*}{1} & \multirow{4}{*}{-} & \multirow{4}{*}{-} & \multirow{4}{*}{134} & \multirow{4}{*}{4.36} & \multirow{4}{*}{12} & \multirow{4}{*}{-} & 0.619 & & \multirow{4}{*}{ DHS } \\
\hline $\mathrm{d}$ & 0.02 & & & & & & & & & 0.626 & 2 & \\
\hline $\mathrm{e}$ & 0.05 & & & & & & & & & 0.612 & & \\
\hline $\mathrm{f}$ & 0.1 & & & & & & & & & 0.605 & & \\
\hline $\mathrm{g}$ & \multirow{3}{*}{0.0} & 0.2 & \multirow{3}{*}{0.5} & \multirow{3}{*}{13} & \multirow{3}{*}{13} & \multirow{3}{*}{134} & \multirow{3}{*}{4.36} & \multirow{3}{*}{12} & 0.060 & 0.602 & $3(\mathrm{a})$ & \multirow{3}{*}{$\begin{array}{l}\text { DL } \\
\text { Uncharged } \\
\text { wall }\end{array}$} \\
\hline $\mathrm{h}$ & & 0.3 & & & & & & & 0.070 & 0.586 & $3(b)$ & \\
\hline $\mathrm{i}$ & & 0.4 & & & & & & & - & - & $3(\mathrm{c})$ & \\
\hline $\mathrm{j}$ & 0.021 & 0.2 & \multirow{4}{*}{0.5} & 14 & 12 & \multirow{4}{*}{134} & \multirow{4}{*}{4.36} & \multirow{4}{*}{12} & 0.058 & 0.606 & 4(a) & \multirow{4}{*}{$\begin{array}{l}\text { DL } \\
\text { Charged } \\
\text { wall }\end{array}$} \\
\hline $\mathrm{k}$ & 0.042 & 0.2 & & 15 & 11 & & & & 0.056 & 0.607 & $4(b), 5(a)$ & \\
\hline 1 & 0.063 & 0.2 & & 16 & 10 & & & & 0.052 & 0.613 & $4(c)$ & \\
\hline $\mathrm{m}$ & 0.063 & 0.3 & & 15 & 11 & & & & 0.056 & 0.608 & $4(d)$ & \\
\hline $\mathrm{n}$ & 0.042 & 0.2 & 0.5 & 30 & 22 & 268 & 6.17 & 12 & 0.057 & 0.607 & $5(\mathrm{~b})$ & Larger \\
\hline o & 0.042 & 0.2 & 0.5 & 21 & 17 & 194 & 4.36 & 17.24 & 0.058 & 0.606 & $5(\mathrm{c})$ & system \\
\hline
\end{tabular}

change between two subsequent configurations depends only on these two configurations. The use of an image sheet per particle is more precise than sheets representing many particles. The additional CPU time can be minimized by polynomial fitting of the time consuming functions in Eqs. (3)(5).

\section{RESULTS AND DISCUSSION}

\section{A. Simulation method}

The NVT MC simulation implemented the usual Boltzmann importance sampling. In an MC step an attempted move was made for each particle in sequential order. Statistical uncertainties were estimated by the block average method where the simulation runs were divided into 10 blocks. The length of the simulations was 4 million $\mathrm{MC}$ steps.

Histograms for distribution functions were accumulated after 10 attempted moves were made for every particle. To evaluate the histogram, the effective range where particles can be located (from $d / 2$ to $H-d / 2$ ) was divided into 200 units of width $(L-d) / 200$. After displacing a particle and before calculating the energies, overlapping was checked by the help of the linked cell method to save CPU time. The linked cell method enables us to check only the neighboring particles for overlap; thus, its demand on CPU time is proportional to only $N$ instead of $N^{2}$. The detailed description of the linked cell method used here can be found in Ref. 34. If an overlap was found, the displacement was refused, and thus the time consuming energy calculation could be avoided. For the maximum displacement of the particles, values in the range $0.1 d \leqslant \Delta r_{\max } \leqslant 0.12 d$ were used. These resulted in acceptance ratios between 0.25 and 0.4 , namely a high percentage of the refusals originated from particle overlap; this made the application of the linked cell method particularly useful.
The state points we consider are related to one of those Zhang et al. ${ }^{30}$ studied in the framework of the SPM model. We kept the system size used by them, as well as the temperature $(300 \mathrm{~K})$, and used similar numbers of particles. The right wall was neutral in all cases. As it was mentioned in Sec. I, we used $d=d_{+}=d_{-}=d_{\mu}=3 \AA$ instead of $4.25 \AA$ that was thought to be too high for the water molecules. The particulars of the simulation parameters are tabulated in Table I. (We did not tabulate every simulation we performed, only those for which profiles are shown. Some of our other simulations are discussed in the text.) The reduced density of the fluid was chosen to be about $\rho^{*}=\left(N_{+}+N_{-}\right.$ $\left.+N_{\mu}\right) d^{3} / W H^{2} \sim 0.7$. Note that in our simulations the cations are the counterions and the anions are the coions, in contrast to the work of Zhang et al., but in this model the two systems are equivalent due to symmetry.

\section{B. Comparison with PM and SPM results}

Our simulation program include the PM and the SPM systems as special cases, thus making it possible to test the program by comparing the results obtained for these systems with those existing in the literature. By using no dipoles $\left(N_{\mu}=0\right)$ and by giving the $\lambda_{i}=1 / \sqrt{\epsilon}$ value to the charging parameter, we obtain a PM system with $\epsilon$ as dielectric constant. For comparison we chose one of the points of Torrie and Valleau ${ }^{13}$ and used the number of ions yielded by their GCMC simulation (sim. $a$ ). This state point corresponds a $0.1 \mathrm{M}$ bulk concentration with the value of $\ln \gamma_{ \pm}=-0.232$ for the activity coefficient. Besides the different ensembles, there are differences in handling the long range corrections, as was discussed in Sec. II. The density profiles compared to those of Torrie and Valleau are shown in Fig. 1(a).

Again, by using $\lambda_{i}=1 / \sqrt{\epsilon}$ for the charging parameter, but neutral hard spheres $\left(\lambda_{\mu}=0\right)$ for the solvent molecules, we get the SPM system. For comparison, one of the state 


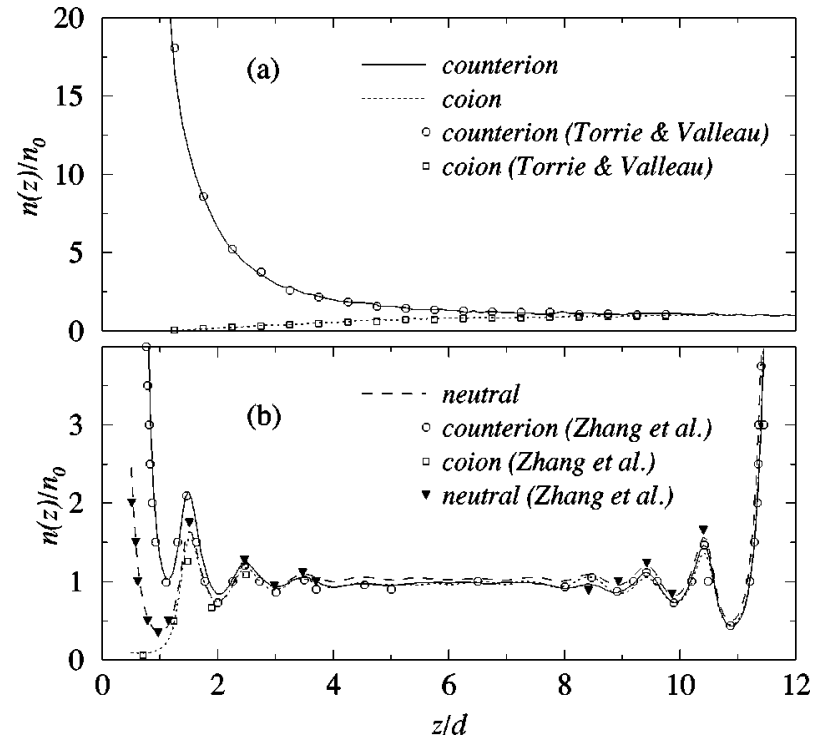

FIG. 1. Normalized density profiles for the PM (a) system compared to the results of Torrie and Valleau (Ref. 13) and for the SPM (b) system with comparison to the data of Zhang et al. (Ref. 30) (a: sim. $a$, b: sim. $b$ ).

points of Zhang et al. ${ }^{30}$ was studied (sim. b). This point corresponds to a 1 molar 1:1 bulk electrolyte with ion activity coefficient $\ln \gamma_{ \pm}=-0.127$ with the value $4.25 \AA$ for the particle diameter on the basis of the procedure described in Sec. I. Comparison of the density profiles can be seen in Fig. 1(b). For a clear correspondence to the results of Zhang et al., we normalized the density profiles with the bulk densities calculated from the 1 molar assumption, $n_{0, i}=N_{A} d^{3}$ $=0.046 \quad$ and $n_{0, \mu}=0.7-2 n_{0, i}=0.608$ with $d=4.25$ $\times 10^{-9} \mathrm{dm}$, as Zhang et al. presumably did. Note that everywhere the densities denoted by $n$ 's are reduced by $d^{3}$.

The bulk densities in Table I are estimated by integrating the density profiles over a range in the middle in the cell where the profiles appeared to be flat. In this way, the calculation of the bulk density is subject to some uncertainty. For consistency, we performed the integration for a range of width of $2 d$ in the middle in every case. Note that the normalization in this way simply means that we divide the absolute density profile by a number about which we think to be approximately the bulk density. In every simulation we normalized the density profiles by the values calculated in this way, except in the cases of simulations $b, h$, and $i$ [Figs. $1(b), 3(b)$, and 3(c)].

It can be seen that for both the PM and the SPM systems agreement is reasonable between the reference data and those obtained from our simulations. In the case of SPM four layers of particles can be observed at both walls. At the charged wall, in every layer there are more coions than counterions. Good agreement with the literature results establishes a consistency between different ensembles (Torrie and Valleau used GC one) and between different methods of estimating long range corrections (Zhang et al. calculated the long range term of the Coulomb potential by a sum using modified Bessel functions).

We comment that the electrode charge densities used by Torrie and Valleau and especially by Zhang et al. are rather high compared to what is experimentally attainable. How-

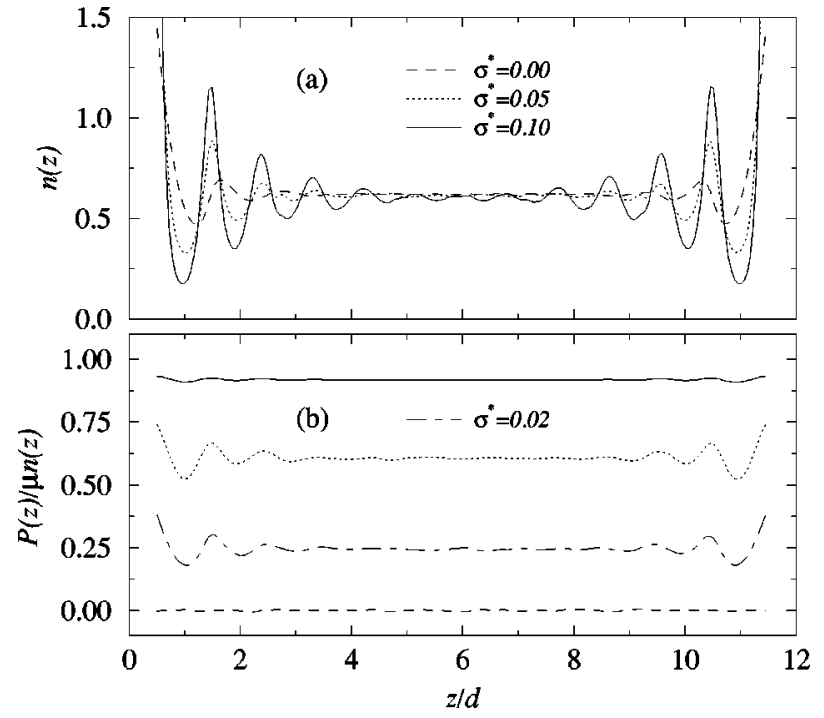

FIG. 2. Number density (a) and polarization/particle (b) profiles for DHS fluid confined between walls carrying $\sigma^{*}$ (left wall) and $-\sigma^{*}$ (right wall) surface charges. (sims. $c-f$ ).

ever, we used these charge densities so that a comparison can be made.

\section{Confined DHS fluid}

There is interest in the structure of water in pores and other confined geometries. The DHS fluid is a very simplified model of water and other polar solvent molecules. For this reason, we investigate the structure of the solvent near charged and uncharged walls. We performed simulations for the same system as listed below for the ion-dipole mixture cases, but without ions, at different surface charges (sims. $c-f$ ). The density profiles are shown in Fig. 2(a), and the polarization per particle profiles in Fig. 2(b). The polarization per particle is obtained by dividing the polarization density by the number density. In Fig. 2(b) the polarization profile is normalized by the dipole moment; thus the value of 1 means a totally polarized fluid. The surface charge densities given in Table I and in Fig. 2 are applied on both walls (positive on the right, negative on the left). It results in a uniform electric field throughout the cell. The density profile for $\sigma^{*}=-0.02$ is omitted in Fig. 2(a) for clarity since it is close to the one at zero charge. According to the symmetry, if we wished, we could average over the profiles at the two walls and obtain a single, presumably more accurate, profile for half the cell.

The results are what one would expect. The density profiles show stronger layering with increasing surface charge. The polarization is zero at zero field, while it is very close to 1 and almost uniform at high surface charge (0.1) showing that the dipoles are strongly oriented. At moderate surface charges a layering can be observed near the walls that reveals that the dipoles are more oriented at the peaks of the density profiles. A possible explanation can be that the particles tend to form chains oriented along the $z$-axis, and at the walls the probability of chain formation may be higher. The particles that are part of a chain are oriented by not only the electric field of the wall, but also by the effect of the low-energy 
configuration of a chain. Note that chains have been found in simulations of bulk DHS fluids. ${ }^{35,36}$

A saturation effect is also produced by the simulations. In Fig. 2(b), it can be seen that the "bulk polarization" (namely the polarization in the center of the cell) increases nonlinearly with the increasing surface charge.

\section{Ion-dipole results for uncharged walls}

At the very beginning in our simulations with full charge and dipole moment it turned out that $\mathrm{MC}$ simulation of the ion-dipole moment is problematic unless we decrease the strengths of the electrostatic forces. If any of the electrostatic interactions is too strong, the system is frozen into lowenergy local configurations. Using the term of Larsen and Rogde,${ }^{33}$ we can say that the simulation is "practically nonergodic."

Therefore we introduced the charging and the polarizing parameter, $\lambda_{i}$ and $\lambda_{\mu}$, and performed several simulations for different pairs of values of these parameters with uncharged walls. We found that if the dipole moment is too strong with respect to the charge $\left(\lambda_{\mu} \geqslant 0.75\right.$ and $\left.\lambda_{i} \leqslant 0.4\right)$, the cations tend to form a chain along the $z$-axis surrounded by appropriately aligned dipoles, while the anions form a similar string separated from the cations. Increasing the charge, the cation- and the anion-string are coming closer to each other and forming pairs of strings. For the influence of additional increase in the charges, the strings break apart and pairs of shorter chains appear, and clusters of ions start to form. If the charge of the ions is very strong, clusters of ions in a latticelike formation appear.

To obtain a solvated bulk electrolyte in the middle of the cell and to get reasonable density profiles for both the ions and the dipoles, both the charge and the dipole moment had to be decreased. We found that for polarizing parameter of $\lambda_{\mu}=0.5$ and for the charging parameter $\lambda_{i} \leqslant 0.3$, "practically ergodic" simulations can be performed. Figure 3 shows the density profiles resulted from three of our simulations. For the sake of comparison the density profiles in Figs. 3(b) and 3(c) are normalized with the bulk densities obtained from the simulation represented by Fig. 3(a) (sim. c). It can be seen that in the cases of $\lambda_{i}=0.2$ and 0.3 the profiles are nearly symmetric and show similar layering to that obtained from the SPM simulation [Fig. 1(b), at the right wall]. Figure 3(a) shows that for $\lambda_{i}=0.2$ the density profiles of the three particles nearly coincide; it implies that the structure of layering is mainly determined by the hard-sphere repulsions. For $\lambda_{i}=0.3$ the bulk density is higher in the middle of the cell [Fig. 3(b)] implying that at this charge the ions are more prone to gather in the middle of the cell and they take place at the wall in a lower probability than in the case of $\lambda_{i}$ $=0.2$. This harmonizes with the results of $\operatorname{Spohr}^{20}$ who has not found contact adsorption in the case of zero surface charge. Figure 3(c) shows an example for a "bad" case where the ions form cluster around $z / d=0.7$.

\section{E. Ion-dipole results for charged walls}

We performed simulations for ion-dipole mixtures near a charged wall at two pairs of $\lambda$ parameters that were found to

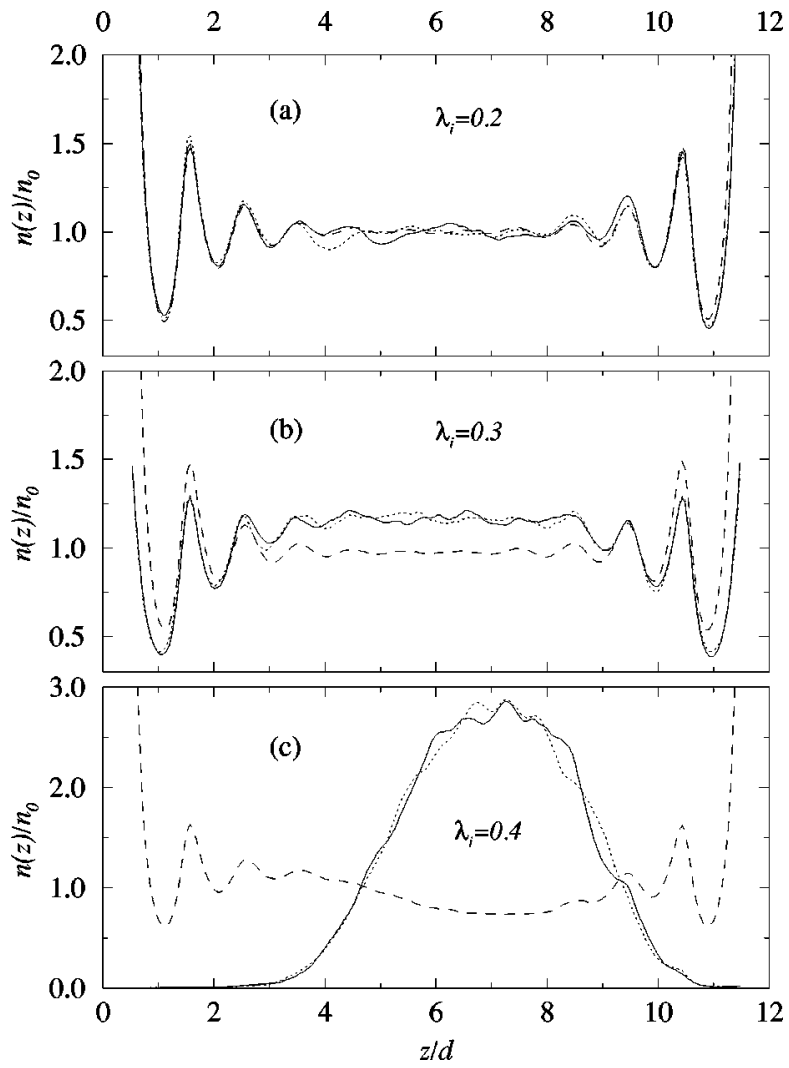

FIG. 3. Normalized density profiles for ion-dipole mixtures confined between uncharged walls with various values of the charging parameter $\lambda_{i}$. Here, and in Figs. 4 and 5, the solid, the dotted and the dashed lines represent cation, anion, and dipole density profiles, respectively (a: sim. $g$, b: sim. $h$, c: $\operatorname{sim} . i)$.

yield "practically ergodic" systems by the zero-charge simulations. The polarizing parameter is fixed at $\lambda_{\mu}=0.5$, while for the charging parameter the values $\lambda_{i}=0.2$ (sims. $j-l)$ and $0.3(\operatorname{sim} . m)$ were used. The density profiles can be seen in Fig. 4.

In the case of $\lambda_{i}=0.2$, the density profiles are basically similar to those obtained from the SPM model. The presence of the solvent molecules induces a strong layering structure at both the charged and the neutral walls. In all cases there are about four layers for every particles. For the lowest surface charge $\left(\sigma^{*}=-0.021\right)$ a considerable residue was found for the coions at the charged wall [Fig. 4(a)]. Increasing the surface charge, this contact adsorption of coions vanishes. In every case, there are more counterions in the second layer than coions, although in the case of the highest surface charge [Fig. 4(c)], the number of anions becomes rather high. This is due to the strong attraction of the cations that are positioned in the first layer at the wall.

Indeed, using a higher charging parameter $\left(\lambda_{i}=0.3\right)$, the attraction of the first layer of cations become even stronger, and a charge inversion occurs in the second layer, namely, the coion density exceeds the counterion one [Fig. 4(d)]. This behavior occurs also in the PM model for higher valence salts, as well as in the SPM model, but the phenomena are less distinct there. Note that we performed a simulation for $\lambda_{i}=0.3$ at an even higher surface charge $\sigma^{*}=-0.095$, and the charge inversion was found to be even stronger. The 


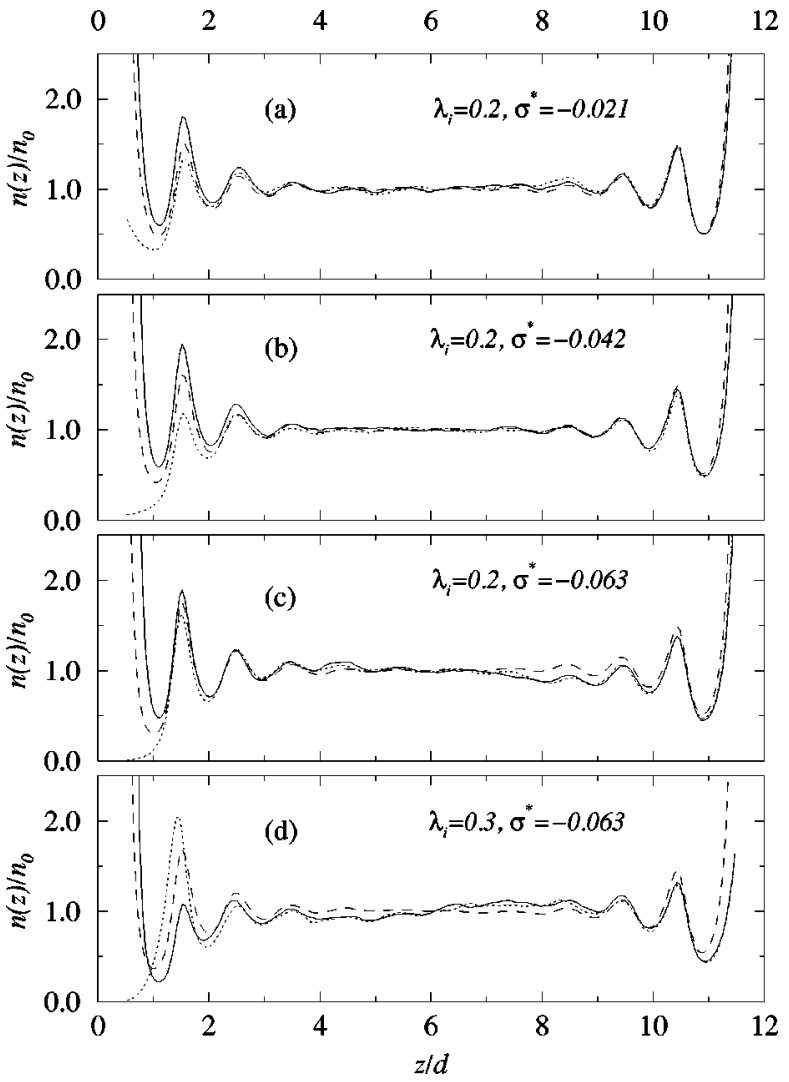

FIG. 4. Normalized density profiles for ion-dipole mixtures with charging parameter $\lambda_{i}=0.2(\mathrm{a}-\mathrm{c})$ and 0.3 (d) for different surface charges on the left wall. For meaning of line types see the caption of Fig. 3 (a: $\operatorname{sim} . j, \ldots$, d: sim. $m)$.

likelihood that this charge inversion is a realistic behavior is strongly supported by the recent simulations of Spohr. ${ }^{20}$ For instance, in the case of positive surface charge, he found a distinct first adsorption layer for $\mathrm{Cl}^{-}$ions, while in the second layer they vanish and $\mathrm{Na}^{+}$ions dominate. Whether charge inversion occurs and its magnitude must depend on the concentration and the kind of the electrolyte, as well as the surface charge.

To investigate the influence of system size on the density profiles, we performed two additional simulations for $\lambda_{i}$ $=0.2$ and $\sigma^{*}=-0.042(\mathrm{sim} . k)$ at larger system sizes. In sim. $n$, the length of the cell was kept fixed $(W=12 d)$, and the area of the wall (and consequently, the number of particles) was increased for twice larger than in sim. $k\left(W^{\prime}\right.$ $=\sqrt{2} W)$. In sim. $o$, the width of the cell was unchanged at $W=4.36 \mathrm{~d}$, while the length of the cell was increased to $H$ $=17.24 \mathrm{~d}$. The necessary numbers of the additional particles were calculated from the bulk densities obtained from sim. $k$ proportionally to the additional volume. However, since the number of particles are integer numbers, the resulting state point may differ somewhat from that of the small system size. Indeed, the density profiles for the long cell [Fig. 5(c)] slightly differ from those of the short cell [Fig. 5(a)]. Nevertheless, in both cases, a definite, although noisy bulk region was obtained in the middle of the cell. Moreover, for the longer cell we experienced poorer statistics at the same length of simulation. From this, we can conclude that the cell length $H=12 d$ seems to be enough to gain reasonable den-

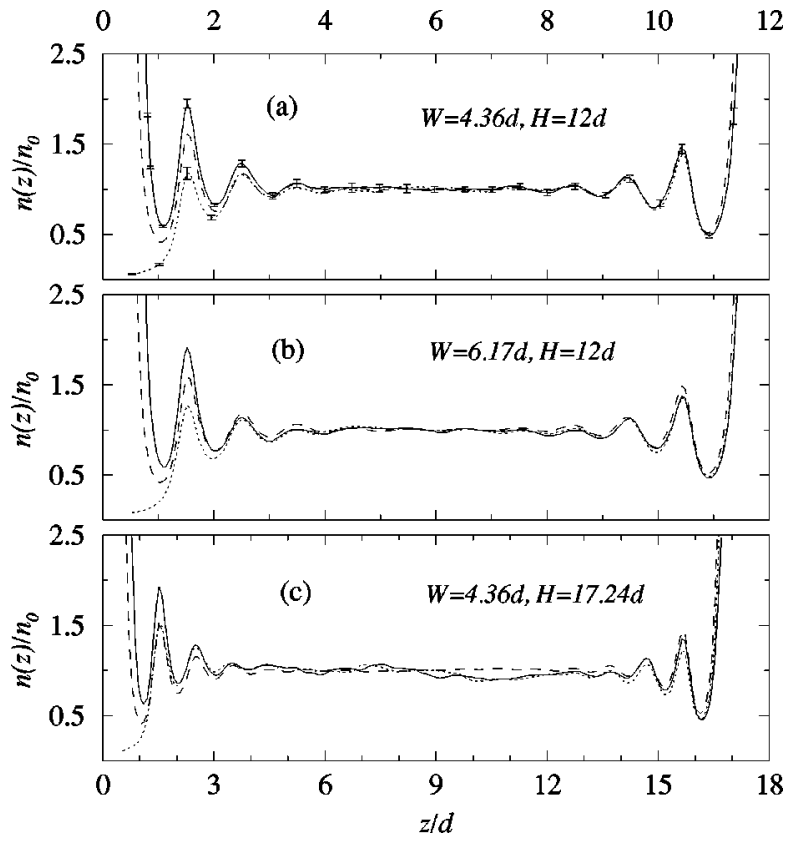

FIG. 5. Normalized density profiles for $\lambda_{i}=0.2$ and $\sigma^{*}=-0.042$ at various system sizes. For meaning of line types see the caption of Fig. 3 (a: sim. $j$, b: $\operatorname{sim} n, \mathrm{c}: \operatorname{sim} . o$ ).

sity profiles in the interfacial regions. Moreover, the profiles for $W=4.36 d$ and $W=6.17 d$ agree very well, which, together with the above conclusion for the cell length, implies that the system size $4.36 d \times 4.36 d \times 12 d$ is appropriate to study the structural features of the DL, at least for this concentration.

Note that we performed simulations at these larger system sizes for the case of uncharged wall also (sim. $g$ ), and similar conclusions could be drawn. Figure 5(a) is identical with Fig. 4(b) except that here error bars are shown for the ionic profiles (the error of the dipole profile is very small). For clarity, in the other figures we do not show the error bars; the orders of magnitude of the statistical uncertainties in the other simulations are similar.

The mean electrostatic potential can be calculated from the equation: ${ }^{18,37}$

$$
\begin{aligned}
\Psi(z)= & \Psi_{i}(z)+\Psi_{\mu}(z) \\
= & 4 \pi \int_{z}^{H}\left[q_{+} n_{+}\left(z^{\prime}\right)+q_{-} n_{-}\left(z^{\prime}\right)\right]\left(z-z^{\prime}\right) d z^{\prime} \\
& -4 \pi \int_{z}^{H} P\left(z^{\prime}\right) d z^{\prime},
\end{aligned}
$$

where $\Psi_{i}$ is the ionic and $\Psi_{\mu}$ is the dipolar contribution to the mean potential, $n_{+}(z)$ and $n_{-}(z)$ are the density distributions of the cation and anion, respectively, and $P(z)$ is the polarization density. Figure 6 shows the reduced potential profiles for simulations $j-m$, where the potentials were reduced by $\Psi^{*}=\beta e \Psi$. For $T=300 \mathrm{~K}, \Psi^{*}=\Psi / 25.9 \mathrm{mV}$. Table II contains the contact values of the reduced potentials (at $d / 2$ ); and their values at $d=0$, namely the potential drops across the interface. The contact values of $\Psi_{i}$ and $\Psi_{\mu}$ were extrapolated by fitting second order polynomials on the logarithms of the three closest values to contact, and then by taking the exponent of the extrapolated function in contact. 


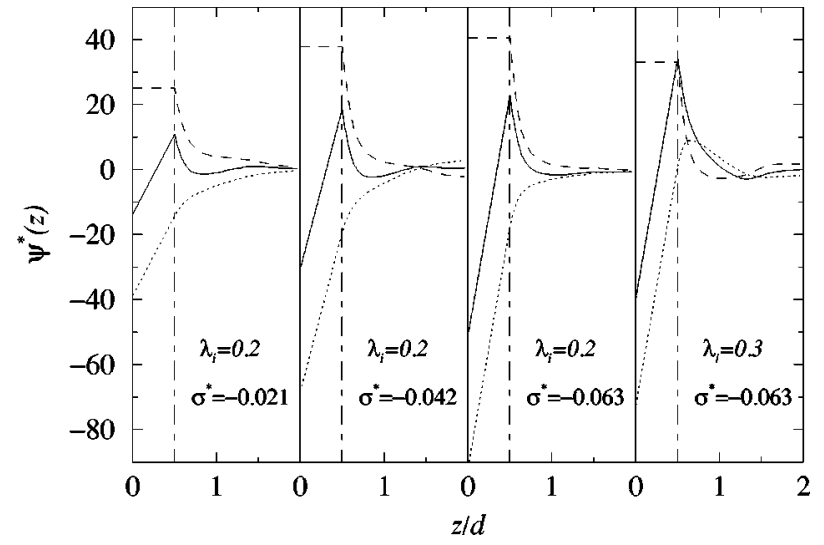

FIG. 6. Reduced mean electrostatic potential profiles for various values of $\lambda_{i}$ and $\sigma^{*}$. The solid, the dotted and the dashed lines represent the total, the ionic and the polarization potentials, respectively (sims. $j-m$ ).

For the ionic part of the potential drop, it stands theoretically that $\Psi_{i}(0)=\Psi_{i}(d / 2)+2 \pi \sigma d$; while for the polarization part $\Psi_{\mu}(0)=\Psi_{\mu}(d / 2)$ since $P(z)=0$ if $0 \leqslant z \leqslant d / 2$. The difference between $\Psi_{i}(0)$ and $\Psi_{i}(d / 2)$ is satisfied exactly in our simulations because of charge neutrality.

The ion-dipole model tends to yield larger potential differences than do either the PM or SPM results. This is encouraging since the PM and SPM models give voltages that are too small, or equivalently capacitances that are too large, unless a layer of low dielectric constant near the wall is postulated. (Table II contains the potential values for the SPM case also for comparison.)

It can be seen that the presence of dipoles manifests itself in an opposite effect in the mean potential. This results in a fluctuation of the total potential profile in which this behavior is a new aspect with respect to the PM and SPM simulations. While the drop from $z=0$ to $d / 2$ in the ionic potential is proportional to the surface charge, the contact potential $\Psi_{i}(d / 2)$ does not seem to depend so strongly on it. This is a consequence of the fact that coions are also present in the DL. That $\Psi_{\mu}(d / 2)$ depends nonlinearly on $\sigma$ implies a saturation behavior for the polarization part of the potential.

Because of the charge inversion, in the case of $\lambda_{i}=0.3$ the potential profiles are quite different (Fig. 6, right side) from those where charge inversion is absent. Not only the total potential, but also the ionic part shows an oscillatory behavior. Moreover, although in a less magnitude, the polarization part also fluctuates. This can be seen more clearly in Fig. 7, which shows the polarization density profiles. Between the first layer of cations and the second layer of anions there is an inversion of orientation in the polarization profile

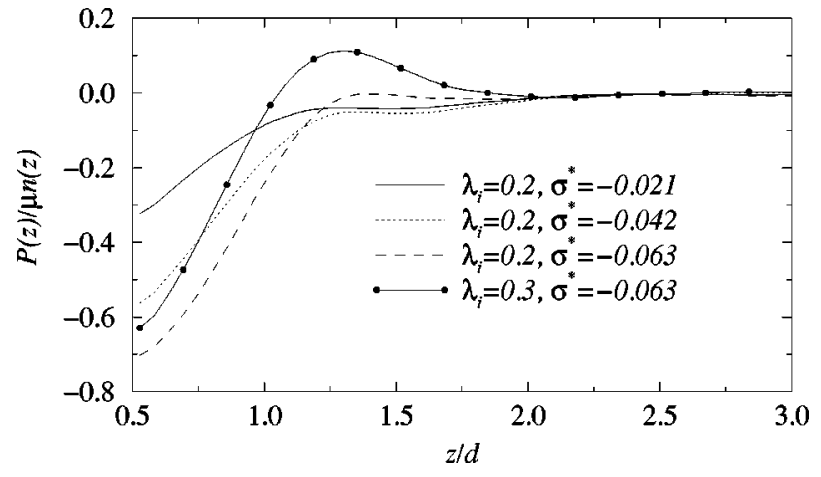

FIG. 7. Polarization density profiles reduced by the number density and the dipole moment for various values of $\lambda_{i}$ and $\sigma^{*}$ (sims. $\left.j-m\right)$.

for $\lambda_{i}=0.3$. From $z=2 d$ the potential profiles decay to zero very rapidly.

Due to the scale of the $y$-axis in Figs. 3-5 it cannot be seen very clearly, but we found a statistical weakness in obeying the mechanical equilibrium along the $z$-axis. In the case of zero surface charge [Figs. 3(a) and 3(b)], this inconsistency manifests itself in an asymmetry in the ionic profiles. Of course, they should be completely symmetrical. To reveal this asymmetry, we calculated the contact values of the density profiles for the ion-dipole simulations (sims. $g-h$ and $j-o$ ) with the method outlined at the section dealing with the mean potentials, and accumulated them in Table III. Comparing the contact values at $d / 2$ and $H-d / 2$, it can be seen that there are some differences between them. This is a consequence of the long extension of the cell in $z$ direction; the particles need more time to travel along the $z$-axis, and more simulation steps are needed to obtain an efficient sampling resulting an ensemble average obeying the condition of mechanical equilibrium in the $z$ direction. This is again the problem of "practical ergodicity.",

While in the case of zero surface charge the symmetry of the profiles offers an obvious method to check the mechanical consistency of the simulation, in the case of charged wall we have to calculate the pressure to check the mechanical equilibrium. According to the contact theorem of nonhomogeneous electrolytes, ${ }^{18,38}$ the pressure of the bulk electrolyte can be calculated from the contact values at a charged wall in the following way:

$$
p=-2 \pi \sigma_{i}^{2}+k T\left(n_{+}^{(i)}+n_{-}^{(i)}+n_{\mu}^{(i)}\right),
$$

where $i$ refers to the wall, and the $n$ 's are the contact values of the densities at the appropriate wall, i.e., $n_{+}^{(1)}=n_{+}(d / 2)$, $n_{+}^{(2)}=n_{+}(H-d / 2)$ and so on. Table III contains the reduced bulk pressures $p^{*}=\beta d^{3} p$ obtained from the contact theorem

TABLE II. Reduced mean electrostatic potentials $\left(\Psi^{*}=\beta e \Psi\right)$ at $z=0$ and at $z=d / 2$.

\begin{tabular}{lccrrrrr}
\hline \hline Simulation & $b$ & $j$ & $k$ & $l$ & $m$ & $n$ & $o$ \\
\hline$\Psi_{i}^{*}(0)$ & -5.95 & -38.9 & -68.8 & -92.1 & -95.7 & -69.5 & -77.1 \\
$\Psi_{i}^{*}(d / 2)$ & -1.53 & -14.3 & -19.7 & -18.5 & -22.0 & -20.4 & -28.0 \\
$\Psi_{\mu}^{*}(d / 2)$ & - & 25.6 & 38.9 & 41.9 & 33.7 & 39.5 & 38.6 \\
$\Psi^{*}(0)$ & -5.95 & -13.3 & -29.9 & -50.3 & -62.0 & -30.0 & -38.5 \\
\hline \hline
\end{tabular}


TABLE III. Contact values of the densities for the ion-dipole simulations. The last two rows contain the reduced pressures $p^{*}=\beta p d^{3}$ of the fluid calculated from the contact theorem at the left $\left(p_{1}^{*}\right)$ and the right $\left(p_{2}^{*}\right)$ wall. The numbers in parentheses denote the statistical uncertainties in the last digit.

\begin{tabular}{lccclllll}
\hline \hline \multicolumn{1}{c}{ Simulation } & $g$ & $h$ & $j$ & \multicolumn{1}{c}{$k$} & \multicolumn{1}{c}{$l$} & \multicolumn{1}{c}{$n$} \\
\hline$n_{+}(d / 2)$ & $0.28(2)$ & $0.10(1)$ & $0.83(3)$ & $2.20(2)$ & $4.85(3)$ & $4.55(3)$ & $2.28(3)$ & $2.45(2)$ \\
$n_{-}(d / 2)$ & $0.27(2)$ & $0.10(1)$ & 0.04 & 0.003 & 0.0 & 0.0 & 0.004 \\
$n_{\mu}(d / 2)$ & $3.49(3)$ & $3.63(2)$ & $3.69(3)$ & $3.94(2)$ & $3.95(3)$ & $4.18(3)$ & $3.87(2)$ & 0.006 \\
$n_{+}(H-d / 2)$ & $0.22(2)$ & $0.10(1)$ & $0.24(1)$ & $0.21(1)$ & $0.18(2)$ & $0.10(1)$ & $0.22(1)$ & $0.18(1)$ \\
$n_{-}(H-d / 2)$ & $0.23(1)$ & $0.10(1)$ & $0.23(1)$ & $0.21(2)$ & $0.20(2)$ & $0.10(1)$ & $0.23(1)$ & $0.24(1)$ \\
$n_{\mu}(H-d / 2)$ & $3.60(3)$ & $3.66(2)$ & $3.54(2)$ & $3.54(3)$ & $3.56(3)$ & $3.57(2)$ & $3.56(2)$ & $3.63(2)$ \\
$p_{1}^{*}$ & $4.04(3)$ & $3.83(2)$ & $4.04(3)$ & $4.08(2)$ & $4.15(3)$ & $4.08(3)$ & $4.09(3)$ & $4.25(3)$ \\
$p_{2}^{*}$ & $4.04(3)$ & $3.86(2)$ & $4.02(2)$ & $3.96(3)$ & $3.93(3)$ & $3.77(2)$ & $4.01(2)$ & $3.99(2)$ \\
\hline \hline
\end{tabular}

at the left $\left(p_{1}^{*}\right)$ and the right $\left(p_{2}^{*}\right)$ wall. Of course, theoretically $p_{1}^{*}=p_{2}^{*}$, but in practice differences due to insufficient sampling regarding a distribution obeying mechanical equilibrium, and statistical uncertainties, may be apparent. It can be seen that in the case of low surface charge ( $\operatorname{sim} . j$ ) the agreement between $p_{1}^{*}$ and $p_{2}^{*}$ is very good. Increasing the surface charge the agreement becomes poorer, and in the cases of the largest inhomogeneity ( $\operatorname{sim} . m$ ) and the long cell (sim. o) the weakest. The latter result shows the weaker statistical efficiency of the simulations in the longer cell, and supports the advice to use as short cell as possible to minimize computer time requirement.

In spite of the differences in the contact values of the densities in the cases of uncharged walls (sims. $g-h$ ), the pressures agree well because they depend on the total contact density that is the same at the two walls, while the composition is different. Thus, we think the equality of pressures in these cases is less meaningful.

Note that the contact theorem of Eq. (14) in the work of Blum and Henderson ${ }^{18}$ differs from that given by Carnie and $\mathrm{Chan}^{37}$ as it is commented in Ref. 38. The difference comes from a different definition of the pressure tensor, and the agreement found here for lower surface charges gives additional support to the definition of Blum and Henderson.

\section{F. Summary}

Canonical MC simulations were performed for a nonprimitive model of electrical DL. The ions are represented with charged, while the dipoles with dipolar hard spheres. To obtain a "practically ergodic" system, both the charge and the dipole moment had to be moderated charging and polarizing parameters. Via these parameters, states equivalent to the PM or SPM have been produced by an "effective" dielectric constant. In addition, molecular mixtures of ions and dipoles with various strengths have been reported.

At larger values of the charging and/or the polarizing parameters, we found clusters and/or strings of particles. This results in an unphysical shape for the profiles. It is possible that extremely long simulations might give better looking profiles. However, this might be due to the clusters moving back and forth rather than to the breaking up the clusters.

The "practical nonergodicity" of the ion-dipole model implies that in the future we will have to consider more sophisticated models of the intermolecular potentials, espe- cially that regarding the water. Nevertheless, even this oversimplified model of the solvent molecules could reveal some interesting phenomena regarding the importance of the solvent molecules in the structure of DL; for instance the charge and the orientation inversion in the second layer.

\section{ACKNOWLEDGMENTS}

The research was supported by the Research Grants Council of Hong Kong (HKU249/95P), by the Hungarian National Research Fund (OTKA-D25516), by CONACyT of Mexico (Grant No. 4186-E9405), by the National Science Foundation (Grant CHE96-01971), and by the donors of the Petroleum Research Fund, administered by the American Chemical Society (Grant No ACS-PRF 31573-AC9). D.H. is a John Simon Guggenheim Memorial Foundation Fellow and is grateful for this support.

${ }^{1}$ D. Henderson and L. Blum, J. Chem. Phys. 69, 5441 (1978).

${ }^{2}$ D. Henderson, L. Blum, and W. R. Smith, Chem. Phys. Lett. 63, 381 (1978).

${ }^{3}$ D. Henderson and L. Blum, J. Electroanal. Chem. 111, 217 (1980).

${ }^{4}$ D. Henderson and M. Lozada-Cassou, J. Colloid Interface Sci. 114, 180 (1986)

${ }^{5}$ S. L. Carnie, D. Y. C. Chan, D. J. Mitchell, and B. W. Ninham, J. Chem. Phys. 74, 1472 (1981).

${ }^{6}$ E. J. Boyle, L. E. Scriven, and H. T. Davis, J. Chem. Phys. 86, 2309 (1987).

${ }^{7}$ L. Mier-y-Teran, S. H. Suh, H. S. White, and H. T. Davis, J. Chem. Phys. 92, 5087 (1990)

${ }^{8}$ Z. Tang, L. E. Scriven, and H. T. Davis, J. Chem. Phys. 97, 9258 (1992).

${ }^{9}$ H. Greberg, R. Kjellander, and T. Åkesson, Mol. Phys. 92, 35 (1997)

${ }^{10}$ L. Guldbrand, B. Jönsson, H. Wennerström, and P. Linse, J. Chem. Phys. 80, 2221 (1984).

${ }^{11}$ B. Svensson, B. Jönsson, and W. E. Woodward, J. Phys. Chem. 94, 2105 (1990).

${ }^{12}$ G. M. Torrie and J. P. Valleau, Chem. Phys. Lett. 65, 343 (1979).

${ }^{13}$ G. M. Torrie and J. P. Valleau, J. Chem. Phys. 73, 5807 (1980).

${ }^{14}$ G. M. Torrie, J. P. Valleau, and G. N. Patey, J. Chem. Phys. 76, 4615 (1982).

${ }^{15}$ W. van Megen and I. Snook, J. Chem. Phys. 73, 4656 (1980).

${ }^{16}$ I. Snook and W. van Megen, J. Chem. Phys. 75, 4104 (1981).

${ }^{17}$ S. L. Carnie and D. Y. C. Chan, J. Chem. Phys. 73, 2949 (1980).

${ }^{18}$ L. Blum and D. Henderson, J. Chem. Phys. 74, 1902 (1981).

${ }^{19}$ G. M. Torrie and G. N. Patey, Electrochim. Acta 36, 1677 (1991) and references cited therein.

${ }^{20}$ E. Spohr, J. Electroanal. Chem. 450, 327 (1998).

${ }^{21}$ M. R. Philpott and J. N. Glosli, in Solid-Liquid Electrochemical Interfaces, Vol. 656 of ACS Symposium Series, edited by G. Jerkiewicz, M. P. Soriaga, K. Uosaki, and A. Wieckowski (ACS, Washington, DC, 1997), Chap. 2, pp. 13-30.

${ }^{22}$ K. Y. Chan, K. E. Gubbins, D. Henderson, and L. Blum, Mol. Phys. 66, 299 (1989). 
${ }^{23}$ W. Y. Lo, K. Y. Chan, and D. Henderson, Mol. Phys. 80, 1021 (1993).

${ }^{24}$ J. M. Caillol, D. Levesque, and J. J. Weis, J. Chem. Phys. 85, 6645 (1986).

${ }^{25}$ J. M. Caillol, D. Levesque, and J. J. Weis, Mol. Phys. 69, 199 (1990).

${ }^{26}$ J. Eggebrecht and P. Ozler, J. Chem. Phys. 93, 2004 (1990).

${ }^{27}$ J. Eggebrecht and G. H. Peters, J. Chem. Phys. 98, 1539 (1993).

${ }^{28}$ Z. Tang, L. E. Scriven, and H. T. Davis, J. Chem. Phys. 97, 494 (1992).

${ }^{29}$ Z. Tang, L. E. Scriven, and H. T. Davis, J. Chem. Phys. 100, 4527 (1994).

${ }^{30}$ L. Zhang, H. T. Davis, and H. S. White, J. Chem. Phys. 98, 5793 (1993).
${ }^{31}$ D. Forciniti and C. K. Hall, J. Chem. Phys. 100, 7553 (1994).

${ }^{32} \mathrm{G}$. Wu and K. Y. Chan (unpublished).

${ }^{33}$ B. Larsen and S. A. Rogde, J. Chem. Phys. 68, 1309 (1978).

${ }^{34}$ D. Boda, K. Y. Chan, and I. Szalai, Mol. Phys. 92, 1067 (1997).

${ }^{35}$ J. J. Weis, and D. Levesque, Phys. Rev. Lett. 71, 2729 (1993).

${ }^{36}$ D. Levesque and J. J. Weis, Phys. Rev. E 48, 5131 (1994).

${ }^{37}$ S. L. Carnie and D. Y. C. Chan, J. Chem. Phys. 74, 1293 (1981).

${ }^{38}$ D. Henderson and L. Blum, J. Chem. Phys. 75, 2025 (1981). 